\title{
Correction to: Convex Analysis and Monotone Operator Theory in Hilbert Spaces
}

Correction to:

H.H. Bauschke, P.L. Combettes, Convex Analysis and Monotone Operator Theory in Hilbert Spaces, CMS Books in Mathematics, https://doi.org/10.1007/978-3-319-48311-5

The original version of this book was inadvertently published without updating the following corrections in Chapters 1-3, 5-13, 16-20, 23, 24, 26, 29, 30, and back matter. These are corrected now.

The updated version of this book can be found at https://doi.org/10.1007/978-3-319-48311-5_1 https://doi.org/10.1007/978-3-319-48311-5_2 https://doi.org/10.1007/978-3-319-48311-5_3 https://doi.org/10.1007/978-3-319-48311-5_5 https://doi .org/10.1007/978-3-319-48311-5_6 https://doi.org/10.1007/978-3-319-48311-5_7 https://doi.org/10.1007/978-3-319-48311-5_8 https://doi.org/10.1007/978-3-319-48311-5_9 https://doi.org/10.1007/978-3-319-48311-5_10 https://doi.org/10.1007/978-3-319-48311-5_11 https://doi.org/10.1007/978-3-319-48311-5_12 https://doi.org/10.1007/978-3-319-48311-5_13 https ://doi.org/10.1007/978-3-319-48311-5_16 https ://doi.org/10.1007/978-3-319-48311-5_17 https ://doi.org/10.1007/978-3-319-48311-5_18 https://doi.org/10.1007/978-3-319-48311-5_19 https ://doi.org/10.1007/978-3-319-48311-5_20 https ://doi.org/10.1007/978-3-319-48311-5_23 https://doi.org/10.1007/978-3-319-48311-5_24 https://doi.org/10.1007/978-3-319-48311-5_26 https://doi.org/10.1007/978-3-319-48311-5_29 https://doi.org/10.1007/978-3-319-48311-5_30 https://doi.org/10.1007/978-3-319-48311-5

(C) Springer International Publishing AG 2019 
For Figures 8.1, 13.1, 16.1, 18.1, 20.1, and 26.1-The usage of font/Symbol $\mathcal{H}$ invariably differs from universally adhered display standards throughout the text content and the same has been incorporated to resolve the issue.

1 Second line after Eq. (1.55): The identity diam $B\left(x_{n} ; \varepsilon_{n}\right)=2 \varepsilon_{n}$ has been replaced by $\operatorname{diam} B\left(x_{n} ; \varepsilon_{n}\right) \leqslant 2 \varepsilon_{n}$.

2 Second line after Eq. (2.17): $n \times n$ has been replaced by $N \times N$.

3 Fact 2.63: The assumption:

let $V$ be a neighborhood of $T x$, and let $R: V \rightarrow \mathcal{K}$. Suppose that $R$ is Fréchet differentiable at $x$ and that $T$ is Gâteaux differentiable at $T x$ has been replaced by:

let $V$ be a neighborhood of $T x$ such that $T(U) \subset V$, and let $R: V \rightarrow \mathcal{K}$. Suppose that $T$ is Gâteaux differentiable at $x$ and that $R$ is Fréchet differentiable at $T x$

4 Exercise 3.2: The phase "a nonempty set" has been replaced by "a nonempty finite set".

5 First line of Exercise 5.9: The phrase "Let $m$ be a strictly positive integer" has been replaced by "Let $m \geqslant 2$ be an integer"

6 Second line after Eq. (6.3): The expression $\sum_{i \in I} \alpha_{i}=1$ has been replaced by $\sum_{i \in I} \alpha_{i} \leqslant 1$.

7 Example 7.10: The assumption "Suppose that $\mathcal{H}$ is finite-dimensional" has been added.

8 Proof of Proposition 8.14(i): The phrase "the convexity of $\phi$ on $C$ " has been replaced by "the convexity of $\phi$ on $I$ ".

9 Example 8.33, last line of the proof: The phrase "so is $\phi=f(\cdot, 1)$ " has been replaced by "so is $\phi^{\diamond}=f(\cdot, 1)$ "

10 Exercise 8.12(ii): The expression $\left|x^{1 / p}+1\right|^{p}$ has been replaced by $-\left|x^{1 / p}+1\right|^{p}$

11 Exercise 8.19: The expression $0 \in C$ has been replaced by $0 \in \operatorname{ri} C$.

12 Equation (9.39): $\mu(\omega)$ has been replaced by $\mu(\Omega)$ 
13 Right-hand side of Equation (9.40): $\mu(\omega)$ has been replaced by $\mu(\Omega)$

14 Proposition 11.8(ii): The expression $C \cap \operatorname{Argmin} f$ has been replaced by $(\operatorname{int} C) \cap \operatorname{Argmin} f$.

15 Definition 12.34: The phrase "it is exact at a point $y \in \mathcal{H}$ " has been replaced by "it is exact at a point $y \in \mathcal{K}$ "

16 Exercise 13.4, Equation (13.39): The expression $\frac{1}{2} u^{2}-|u|-\frac{1}{2}$ has been replaced by $\frac{1}{2} u^{2}+|u|-\frac{1}{2}$.

17 Proposition 13.24 and Corollary 13.25: The assumption "Let $\mathcal{K}$ be a real Hilbert space" has been added.

18 Proof of Proposition 13.45: The phrase "in view of Proposition 13.13 " has been removed.

19 In Eq. (13.26): The expression $\sup _{x_{i} \in \mathcal{H}}$ has been replaced by $\sup _{x_{i} \in \mathcal{H}_{i}}$.

20 Equation (17.45): The expression $N_{C} x \cap B\left(0 ; \phi^{\prime}\left(d_{C}(x)\right)\right)$ has been replaced by $N_{C} x \cap B\left(0 ; \phi^{\prime}(0)\right)$.

21 Corollary 18.20, first sum in Eq. (18.40): The expression $\sum_{i=1}^{m} \alpha_{i} \operatorname{Prox}_{f_{i}}$ has been replaced by $\sum_{i \in I} \alpha_{i} \operatorname{Prox}_{f_{i}}$

22 Fourth line after Eq. (18.40): The expression $\nabla f=\sum_{i \in \mathcal{H}} \alpha_{i} \operatorname{Prox}_{f_{i}}$ has been replaced by $\nabla f=\sum_{i \in I} \alpha_{i} \operatorname{Prox}_{f_{i}}$.

23 Exercise 20.1(iii): The phrase "is differentiable" has been replaced by "is convex and differentiable".

24 Equation (24.81): The expression

$$
\xi \mapsto \begin{cases}\xi \operatorname{arctanh}^{-1}(\xi)+\frac{1}{2}\left(\ln \left(1-\xi^{2}\right)-\xi^{2}\right), & \text { if }|\xi|<1 ; \\ +\infty, & \text { if }|\xi| \geqslant 1 .\end{cases}
$$

has been replaced by

$$
\xi \mapsto \begin{cases}\frac{(1+\xi) \ln (1+\xi)+(1-\xi) \ln (1-\xi)-\xi^{2}}{2}, & \text { if }|\xi|<1 ; \\ \ln (2)-1 / 2, & \text { if }|\xi|=1 ; \\ +\infty, & \text { if }|\xi|>1 .\end{cases}
$$


25 Example 24.51: The expression "is a proximal thresholder" has been replaced by "If $\mathcal{H}=\mathbb{R}$, then $\operatorname{Prox}_{f}$ is a proximal thresholder".

26 Exercise 24.6: The phrase "Show that $(\forall x \in \mathcal{H})$ " has been replaced by "Show that $(\forall x \in \mathbb{R})$ "

27 Corollary 26.6: The statement:

Then $x \in \operatorname{zer}(A+B), u \in \operatorname{zer}\left(-A^{-1} \circ(-\mathrm{Id})+B^{-1}\right),(x,-u) \in \operatorname{gra} A$, and $(x, u) \in \operatorname{gra} B$

has been replaced by:

Then $x \in \operatorname{zer}(A+B),-u \in \operatorname{zer}\left(-A^{-1} \circ(-\mathrm{Id})+B^{-1}\right),(x, u) \in \operatorname{gra} A$, and $(x,-u) \in \operatorname{gra} B$

28 Theorem 26.34, line after Eq. (26.97): The inclusion $\gamma \in$ ] $0,1 /\|L\|^{2}[$ has been replaced by $\gamma \in] 0,1 /\|L\|[$

29 Statement of Proposition 29.4: The identity $\boldsymbol{C}=\chi_{i \in I} C_{i}$ has been replaced by $\boldsymbol{C}=\mathcal{H} \cap \chi_{i \in I} C_{i}$

30 Exercise 29.23: The statement "Show that $(\forall x \in \operatorname{dom} f \backslash C)$ " has been replaced by "Show that $(\forall x \in \operatorname{dom} f \backslash C) d_{C}(x) \leqslant\left\|x-x_{0}\right\|(f(x)-$ $\xi) /\left(f(x)-f\left(x_{0}\right)\right) . "$

31 Example 29.47: The expression $\left.\left.C=\operatorname{lev}_{\leqslant 0} f=\right]-\infty,-1 / 2\right]$ has been replaced by $\left.\left.C=\operatorname{lev}_{\leqslant 0} f=\right]-\infty,-1\right]$.

32 Equation (30.8) and the sentence before:

and therefore that

$$
\left(x_{n+1}-T x_{n}\right)_{n \in \mathbb{N}} \text { is bounded. }
$$

has been replaced by

and therefore, in view of (i), that

$$
\left\|x_{n+1}-T x_{n}\right\|=\lambda_{n}\left\|x-T x_{n}\right\| \rightarrow 0 .
$$

